\title{
CONTRIBUTION TO KNOWLEDGE ON THE VARIABILITY OF AROLLA PINE WOODLANDS OF THE NORTH-EASTERN TATRA MOUNTAINS
}

\author{
Peter KUČERA ${ }^{1}$, Peter BARANČOK ${ }^{2}$ \\ ${ }^{1}$ Comenius University in Bratislava, Botanical Garden, workplace Blatnica, Blatnica 315, \\ SK-038 15 Blatnica pri Martine, Slovakia \\ ${ }^{2}$ Slovak Academy of Sciences, Institute of Landscape Ecology \\ e-mail: peter.kucera@uniba.sk
}

\begin{abstract}
Knowledge of the overall syntaxonomic and habitat variability of forest communities with Arolla pine (Pinus cembra) within the Tatra Mountains (Western Carpathians) is still insufficient as field research was hindered by their hardly accessible localities and deforestation for high mountain grazing. Arolla pine woodlands were traditionally classified within the association Pino cembrae-Piceetum Myczkowski et Lesiński 1974, but recent surveys recognize more numerous units. Hitherto unpublished relevés from the north-eastern part of the Tatra Mountains document the occurrence of several floristically and ecologically distinct Arolla pine communities: (1) acid woodland of Homogyno alpinae-Pinetum cembrae on nutrient-poor habitats over quartzitic bedrock, and (2) calcareous woodlands of species-poor Pyrolo rotundifoliae-Pinetum cembrae of sites with a well-developed, tangled humus soil horizon, Cystopterido montanae-Pinetum cembrae on habitats influenced by both limestones and quartzites, Primulo elatioris-Pinetum cembrae bound to sites with the most favourable humidity, and Seslerio tatraePinetum cembrae with the most pronounced calcareous character.
\end{abstract}

Keywords: Belianske Tatry Mts, phytocoenology, Pinus cembra, Slovakia, syntaxonomy, Vysoké Tatry Mts, Western Carpathians.

\section{Introduction}

The current native occurrence of Arolla pine in the Western Carpathians is exclusively bound to the Tatra Mountains, although the species is found in other mountain ranges [29, 8]. Due to centuries-long historical land management (forest and krummholz exploitation and burning for high mountain grazing) the species' distribution was significantly reduced in this highest mountain range of the Carpathians up to the period 1900-1950. Long-term human impact on the Tatra's Arolla pine and mixed Arolla pine forest stands had resulted in a decrease of the extent of their natural distribution to isolated islands which have been preserved mostly within the High Tatras, either in their northern (Poland/Slovakia) or southern (Slovakia) part.

The first phytocoenological relevé of Pinus cembra woodland from the Tatra Mountains was published in the classic study of Pawłowski et al. [32]. Due to a very limited distribution of Arolla pine forests within the Polish Tatras, only a few later phytocoenological studies were carried out [i.e. 30, 38, 39].

More numerous studies with documentation of Arolla pine woodlands were published in Slovakia: for the overall literature review see Kučera [17]. Within the territory lying to the north of the main ridge of the Tatra Mountains, the first Arolla pine relevés were published by Samek et al. [33]. They were followed by Zlatník [40]. Barančok \& Varšavová [2] comprehensively carried out a study on the forests of the Bielovodská dolina Valley. Kukla et al. [20] also published a 
record from the same region. Floristically special Arolla pine plant communities were documented in the monographic vegetation survey of forests of the Belianske Tatry Mts elaborated by Kanka [12].

The most recent data on Arolla pine phytocoenoses within the northern High Tatras were published by Jasík et al. [11] and Jasík \& Dítě [10], who documented findings of Linnaea borealis, an extremely rare species in the Western Carpathians. Finally, Zięba et al. [39] published a larger number of Arolla pine relevés from both the Slovakian northern and, especially, southern part of the Tatra Mountains.

The aim of this paper is to contribute to knowledge of phytocoenological variability of the Arolla pine woodlands of the Tatra Mountains and to make access to hitherto unpublished relevés from the Slovakian north-eastern part of the High Tatras. First, a general overview is given of previously published classifications of the plant communities of the Tatras with Pinus cembra.

Outline of the approaches to syntaxonomic classification of Arolla pine woodlands of the Tatra Mountains

Classifications of Arolla pine and mixed Arolla pine-Norway spruce plant communities in Slovakia and Poland were considerably influenced by Myczkowski and Lesiński [30], who delimited the respective forest communities into the association Cembro-Piceetum Myczkowski et Lesiński 1974, later mostly known under the altered name "Pino cembrae-Piceetum". ${ }^{1}$ Although some Polish authors do not recognize Arolla pine phytocoenoses as a separate forest plant community [24, 25, 26, 22, 23, 27], the most frequent approach was to assign all Pinus cembra phytocoenoses recorded within the Tatras to that association.

The association name Cembro-Piceetum Myczkowski et Lesiński 1974 was also applied in the first comprehensive classification of the Arolla pine phytocoenoses related to the Tatra Mountains (see [1]). On the contrary, Wojterska et al. [38] assigned their relevés to the association Larici-Cembretum Ellenberg 1963 nom. inval. (= Larici-Pinetum cembrae Ellenberg et Klötzli 1974 nom. cons. propos. [see 16, 17]); however, this plant community is phytochorologically restricted to the Alps.

Kanka [12, cf. 13] published Arolla pine relevés classified within two different communities: Cembro-Piceetum calamagrostietosum variae Barančok ex Kanka 2008 nom. inval. (Art. 3i, 3o, 14b) and Vaccinio myrtilli-Piceetum pinetosum cembrae Kanka 2008 nom. inval. (Art. 3o). The overall phytocoenotic variability of natural Arolla pine woodlands in Slovakia was differentiated by Kučera [15] into three different forest communities.

Subsequently, Kučera published a revised classification of Arolla pine plant communities of the Tatra Mountains [16]. In contrast to the previous authors, calcicolous and acid (silicicolous) phytocoenoses are there split between two orders of the class Vaccinio-Piceetea Br.-B1. in Br.-Bl. et al. 1939 and the fundamental habitat and floristic differences are classified within more numerous floristically and ecologically distinct associations:

Piceetalia abietis Pawłowski ex Pawłowski et al. 1928 nom. corr.

Homogyno alpinae-Pinion cembrae P. Kučera 2017

1. Homogyno alpinae-Pinetum cembrae P. Kučera 2017

1 The originally used form Cembro-Piceetum is nomenclaturally correct (Art. 14b of the Code), therefore it should be preferred above the later changed name, Pino cembrae-Piceetum. 
2. Prenantho purpureae-Pinetum cembrae P. Kučera 2017

3. Mylio taylorii-Pinetum cembrae P. Kučera 2017

4. Cembro-Piceetum Myczkowski et Lesiński 1974

Cortuso matthioli-Piceetalia abietis P. Kučera $2022^{2}$

Calamagrostio variae-Pinion cembrae P. Kučera 2017

5. Seslerio tatrae-Pinetum cembrae P. Kučera 2017

6. Cystopterido montanae-Pinetum cembrae P. Kučera 2017

Zięba et al. [39] published very numerous and valuable relevé data of Arolla pine forests classified into associations Vaccinio-Cembretum (Pallmann et Hafter 1933) Oberdorfer 1962 nom. inval. [cf. 16] and Swertio perennis-Pinetum cembrae Zięba et al. 2018 with a further syntaxonomical subdivision. This vegetation survey was discussed and commented on in detail by Kučera [17].

\section{Material and Methods}

The field research was performed by P. Barančok in the north-eastern territory of the Tatra Mountains in the years 1993-1998 [see also 2]. Cover-abundance values and sociability were recorded following Braun-Blanquet [4]. Although the phytocoenological relevés from the years 1994-1995 were used for an older synthesis [1], they were never published - except for the relevés from the Belianske Tatry Mts [see 12: tab. 11, rels 1-4]. Therefore they could not be included in the comprehensive syntaxonomic classification of the Tatra Mountains' Pinus cembra woodlands [16]. Hence all of them are here re-published for better recognition of phytocoenological diversity of calcareous Arolla pine phytocoenoses found in the Tatra Mountains. However, the species composition of the individual relevés is not complete: data on mosses and lichens are omitted. The respective indetermined field samples (mostly mosses, but also several lichens and phanerogames) are stored in the second author's collection.

The relevés were previously electronically stored using the Turboveg for Windows database software [6] and submitted to Slovak phytoceonological database ([34], http://www.givd.info/ID/EU-SK-001). For this paper, the respective relevés were revised and partially corrected using a current Turboveg version [5]. Turboveg relevés were subsequently exported for table processing within the software packages JUICE [36, 37].

Selection of differential species is made with consideration of preferential species groups of previously published classifications of Arolla pine communities [12, 16, 39].

Plant taxa names follow the checklist of Marhold et al. [21]. Syntaxonomical nomenclature is adjusted according to the recently published phytocoenological Code [35]. Bedrock types for the individual relevé sites are given according to map of Nemčok et al. [31]. Common soil names are supplemented with WBR 2006 names given according to the reference table in [28].

\section{Results and Discussion}

Phytocoenoses of the surveyed area are grouped into two different ecologically and

\footnotetext{
2 Kučera [16] originally used the name Athyrio filicis-feminae-Piceetalia Hadač ex Hadač et al. 1969. Due to multiple syntaxonomic and nomenclatural problems associated with pseudonymical application of the validly published name Athyrio-Piceetalia Hadač 1962 [see 18], a new order name Cortuso-Piceetalia for calcicolous communities of the class Vaccinio-Piceetea Br.-BI. in Br.-BI. et al. 1939 is formally proposed [19].
} 
floristically units, evaluated at the rank of an alliance: silicicolous Homogyno alpinae-Pinion cembrae and calcicolous Calamagrostio variae-Pinion cembrae [cf. 16].

\section{Homogyno alpinae-Pinion cembrae P. Kučera 2017}

Communities of this alliance are within the Tatra Mountains distributed in the areas formed by granitoid and other non-carbonate rocks. Habitats are usually very nutrient-poor, which is reflected in their species-poor composition. Vaccinium myrtillus, Avenella flexuosa, Calamagrostis villosa, Vaccinium vitis-idaea are the characteristic and most frequent species of the field layer, accompanied by widely distributed species of the forests of higher montane altitudes, e.g. Oxalis acetosella, Homogyne alpina, Dryopteris dilatata, Luzula sylvatica and Gentiana asclepiadea. The alliance is represented only by the association Homogyno alpinaePinetum cembrae within the relevé data presented here.

\subsection{Homogyno alpinae-Pinetum cembrae P. Kučera 2017 (Tab. 1, rels. 1-2)}

This Arolla pine woodland comprises the most species-poor phytocoenoses. They differentiate mostly negatively, by absence of species typical for other associations of the alliance Homogyno alpinae-Pinion [16]. The field layer is dominated by either Vaccinium myrtillus or Dryopteris dilatata, less frequently by Avenella flexuosa. More nutrient-demanding Prenanthes purpurea, Adenostyles alliariae, Senecio nemorensis agg. and Veratrum album ssp. lobelianum are infrequent and do not reach higher cover. In ecologically suitable sites are present Huperzia selago and even Gentiana punctata.

The documented relevés in the Kolová dolina Valley belong to the subassociation Homogyno alpinae-Pinetum cembrae typicum. A similar community was recorded there also by Zięba et al. [39] [cf. 17].

Association Homogyno alpinae-Pinetum cembrae is usually mistaken for the association Cembro-Piceetum Myczkowski et Lesiński 1974. The latter mixed Arolla pine community has ecologically special habitat facilitating the presence of species populations of Empetrum nigrum agg. (most probably E. hermaphroditum), Vaccinium gaultherioides and Listera cordata, as well as very high cover of Pinus mugo. The recorded presence of Abies alba in the tree layer is remarkable.

The other associations of the alliance Homogyno alpinae-Pinion cembrae differentiate by either (1) species-rich ground layer with Lophozia ventricosa and Mylia taylori, Mylio taylorii-Pinetum cembrae, or (2) higher herb species diversity, Prenantho purpureae-Pinetum cembrae with stands occupying less frequently but considerable more nutrient-rich habitats on granitic rocks [16].

\section{Calamagrostio variae-Pinion cembrae P. Kučera 2017}

Arolla pine forest communities of this alliance occupy soils developed over various carbonate rocks. They are positively differentiated by the presence of basiphilous species, usually calciphytes, i.e. Asplenium viride, Calamagrostis varia, Cirsium erisithales, Cortusa matthioli, Sesleria tatrae. The second significant feature is the presence of multiple species growing within the supramontane coniferous forests of the Western Carpathians almost exclusively in the order Cortuso-Piceetalia: Geranium sylvaticum, Phyteuma spicatum, Polygonatum verticillatum, Valeriana tripteris, Viola biflora etc. Potential sporadic occurrence of some of these species in the 
phytocoenoses of the order Piceetalia abietis Pawłowski ex Pawłowski et al. 1928 nom. corr. reliably indicates ecologically special rare habitats.

Due to the much smaller total area of carbonate rocks within the Tatras [31] and extensive historical deforestation for high mountain grazing [7], frequently only small groups or individuals of Pinus cembra were found, often only young trees [3].

In the surveyed area, the alliance is represented by the associations Pyrolo rotundifoliaePinetum cembrae, Cystopterido montanae-Pinetum cembrae and Primulo elatioris-Pinetum cembrae.

\subsection{Pyrolo rotundifoliae-Pinetum cembrae ass. nov. prov. (Tab. 1, rel. 3)}

Rel. 3 with its species-poor phytocoenosis characterizes a transitional community towards the alliance Homogyno alpinae-Pinion cembrae. The ecological impact of calcareous rocks is significantly blocked by a thick layer of organic horizon originated under the considerable influence of fallen plant litter (mostly needles of Pinus mugo, also Pinus cembra) while the process of natural vegetation succession is substantially blocked by the special orographic situation. Under such habitat conditions evolves a special type of rendzina (histic rendzic leptosol) with tangel humus formation [cf. 28, 14], which is also present at the site of rel. 3 .

Plant community of the habitat is characterized by acidophytes as dominating species, for example in rel. 3, Avenella flexuosa and Vaccinium vitis-idaea, or by generally low cover of the field layer. Differential species of the alliance Calamagrostio variae-Pinion cembrae are present in reduced number and abundances, in the rel. 3 are present only Calamagrostis varia and Pyrola rotundifolia.

A similar species-poor community was documented by Zięba et al. [39: suppl. S2, rel. 52] [cf. 17: tab. 1]; however, it resembles the association Cembro-Piceetum Myczkowski et Lesiński 1974 due to the co-occurrence of Vaccinium gaultherioides and Empetrum hermaphroditum. Influence of the carbonate bedrock is limited only to the sporadic presence of Sesleria tatrae.

\subsection{Cystopterido montanae-Pinetum cembrae P. Kučera 2017 (Tab. 1, rels. 4-6)}

(syntax. syn. Swertio perennis-Pinetum cembrae Zięba et al. 2018)

The characteristic attribute of this community is the mix of abundant acidophytes (Vaccinium myrtillus, Avenella flexuosa, V. vitis-idaea), calciphytes (Cortusa matthioli, Asplenium viride) and the group of nutrient-demanding species (Geranium sylvaticum, Phyteuma spicatum, Valeriana tripteris, Viola biflora etc.) (cf. Table 1 and Zięba et al. [39: suppl. S3, "var. Valeriana tripteris"]).

The habitat of the Arolla pine stands recorded in the Kolová dolina Valley is characterized by shared ecological influence of limestone and non-carbonate rocks (quartzites). Resulting impact on the species composition and structure is reflected by the absolute dominance of Vaccinium myrtillus and by the presence of Calamagrostis villosa, reaching higher cover values as well. The community is similar to Cystopterido montanae-Pinetum cembrae typicum [cf. 16,]; rel. 5 from the Kolová dolina, having higher cover of Adenostyles alliariae, indicates a transitional community towards the association Primulo-Pinetum cembrae.

Except for the localities presented here from the High Tatras, one stand of the subassociation Cystopterido montanae-Pinetum cembrae typicum was recently recorded in the Belianske Tatry Mts by Zięba et al. [39]. 
Table 1: Floristic differentiation of recorded Arolla pine phytocoenoses in the Kolová dolina Valley, Monkova dolina Valley and in the Sika Valley

Column 1-2: Homogyno alpinae-Pinetum cembrae

Column 3: Pyrolo rotundifoliae-Pinetum cembrae ass. prov.

Column 4-6: Cystopterido montanae-Pinetum cembrae

Column 7-10: Primulo elatioris-Pinetum cembrae

Column 11-15: Seslerio tatrae-Pinetum cembrae

Absent values (*) are given within descriptions of relevé localities.

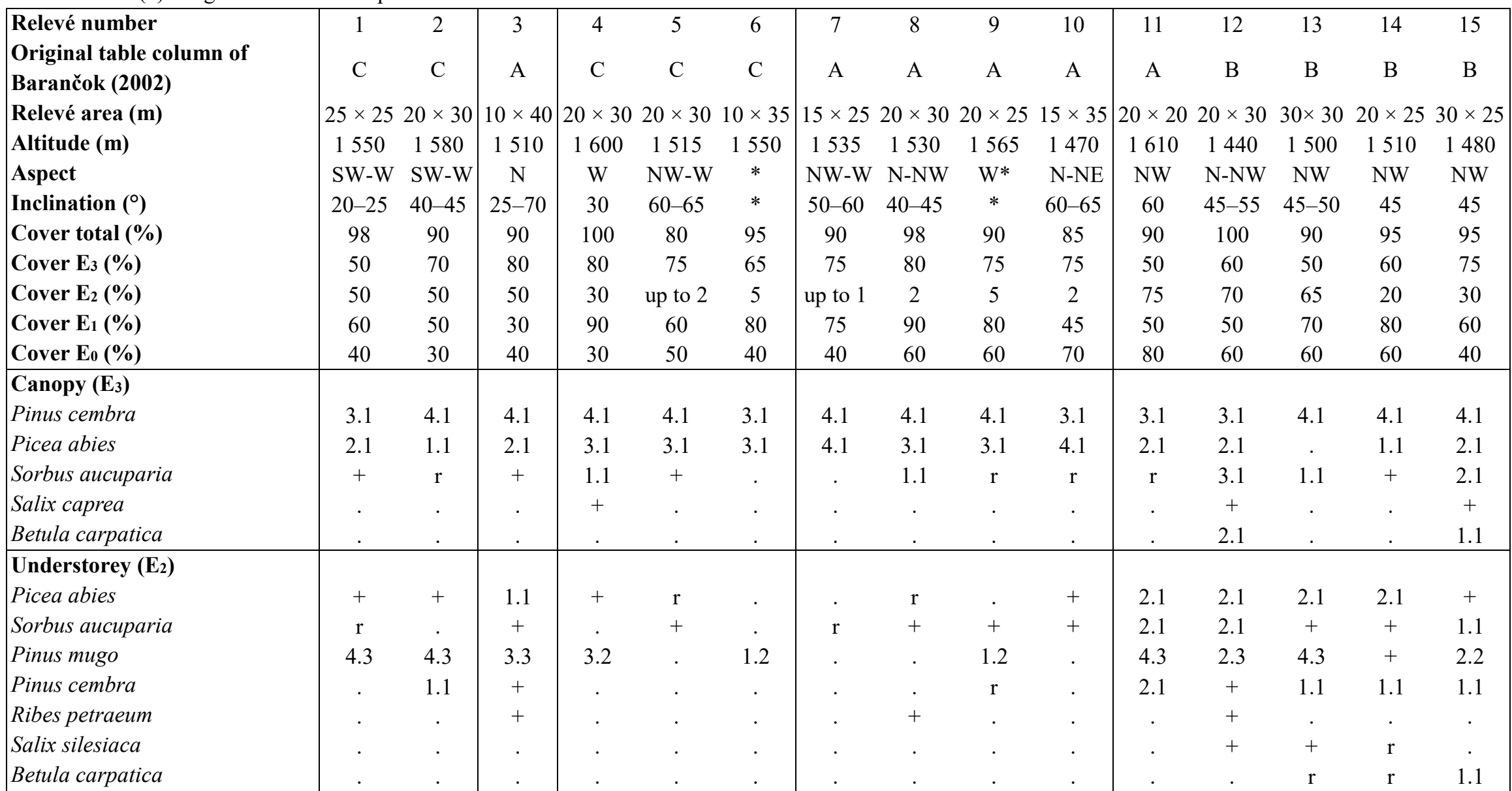




\begin{tabular}{|c|c|c|c|c|c|c|c|c|c|c|c|c|c|c|c|}
\hline Relevé number & 1 & 2 & 3 & 4 & 5 & 6 & 7 & 8 & 9 & 10 & 11 & 12 & 13 & 14 & 15 \\
\hline Lonicera nigra & . & . & . & . & . & . & . & . & . & . & . & 1.1 & . & $\mathrm{r}$ & . \\
\hline Daphne mezereum & . & . & . & . & . & . & . & . & . & . & . & . & . & $\mathrm{r}$ & . \\
\hline Salix caprea & . & . & . & . & . & . & . & . & . & . & . & . & . & . & + \\
\hline \multirow{2}{*}{\multicolumn{16}{|c|}{$\begin{array}{l}\text { Field layer }\left(E_{1}\right) \\
\text { Young trees and shrubs }\end{array}$}} \\
\hline & & & & & & & & & & & & & & & \\
\hline Sorbus aucuparia & $\mathrm{r}$ & $\mathrm{r}$ & + & 1.1 & 1.1 & + & 1.1 & 1.1 & 1.1 & + & 1.1 & 1.2 & + & + & 1.1 \\
\hline Picea abies & . & . & $\mathrm{r}$ & . & + & $\mathrm{r}$ & . & $\mathrm{r}$ & . & $\mathrm{r}$ & . & . & 1.1 & . & + \\
\hline Ribes petraeum & . & . & . & . & . & . & + & + & . & $\mathrm{r}$ & . & + & $\mathrm{r}$ & + & $\mathrm{r}$ \\
\hline Salix sp. & . & . & . & $\mathrm{r}$ & . & $\mathrm{r}$ & . & . & . & . & + & . & + & + & . \\
\hline Pinus cembra & . & $\mathrm{r}$ & $\mathrm{r}$ & . & . & $\mathrm{r}$ & . & . & . & . & . & . & . & + & + \\
\hline Daphne mezereum & . & . & . & . & . & . & . & . & $\mathrm{r}$ & . & . & $\mathrm{r}$ & $\mathrm{r}$ & $\mathrm{r}$ & $\mathrm{r}$ \\
\hline Lonicera nigra & . & . & . & . & $\mathrm{r}$ & . & $\mathrm{r}$ & . & . & . & . & . & $\mathrm{r}$ & . & + \\
\hline Salix silesiaca & . & . & . & . & . & . & . & . & . & . & . & + & . & . & . \\
\hline Betula carpatica & . & . & . & . & . & . & . & . & . & . & . & . & . & + & . \\
\hline Salix caprea & . & . & . & . & . & . & . & . & . & . & . & . & . & . & + \\
\hline Rosa pendulina & . & . & . & . & . & . & . & . & . & . & . & . & . & . & $\mathrm{r}$ \\
\hline \multicolumn{16}{|l|}{ Differential species } \\
\hline Calamagrostis villosa & 2.2 & $\mathrm{r}$ & . & $3.3^{\#}$ & $1.2^{\#}$ & $3.2^{\#}$ & . & . & . & . & . & . & . & . & . \\
\hline Gentiana punctata & 1.1 & $\mathrm{r}$ & . & . & . & . & $\mathrm{r}$ & . & . & $\mathrm{r}$ & . & . & . & . & . \\
\hline Solidago virgaurea &. & . & . & $\mathrm{r}$ & $\mathrm{r}$ & $\mathrm{r}$ & $\mathrm{r}$ & . & . & + & . & . & . & . & . \\
\hline Prenanthes purpurea & . & . & . & . & 1.1 & $\mathrm{r}$ & . & . & . & . & . & . & . & . & + \\
\hline Chrysosplenium alternifolium & . & . & . & . &. & . & . & + & $\mathrm{r}$ & $\mathrm{r}$ & . & . & . & . & . \\
\hline Primula elatior subsp. tatrensis & . & . & . & . & . & . & . & 1.1 & + & . & . & . & . & . & . \\
\hline Stellaria nemorum & . & . & . & . & . & . & . & +.2 & +.2 & . & . & . & . & . & . \\
\hline Thalictrum aquilegiifolium & . & . & . & . & . & . & . & + & + & . & . & + & . & . & . \\
\hline Myosotis alpestris & . & . & . & . & . & . & . & + & $\mathrm{r}$ & . & . & . & + & . & . \\
\hline Doronicum austriacum & . & . & . & . & . & . & 1.2 & 1.2 & . & . & . & 1.2 & . & . & . \\
\hline Cardaminopsis halleri & . & . & . & $\mathrm{r}$ & . & . & . & $\mathrm{r}$ & $\mathrm{r}$ & . & . & . & . & . & . \\
\hline Chaerophyllum hirsutum & . & . & . & . & . & . & . & 2.2 & . & . & . & . & . & . & . \\
\hline Luzula luzuloides & +.2 & . & . & . & . & . & 2.2 & . & +.2 & + & + & 1.2 & . & . & . \\
\hline Bartsia alpina &. & . & . & . & . & . & . & . &. & . & $\mathrm{r}$ & . & +.2 & + & . \\
\hline Ranunculus alpestris & . & . & . & . & . & . & . & . & . & . & $\mathrm{r}$ & . & + & + & . \\
\hline Saxifraga aizoides & . & . & . & . & . & . & . & . & . & . & $\mathrm{r}$ & . & . & + & . \\
\hline Hedysarum hedysaroides & . & . & . & . & . & . & . & . & . & . & . & $\mathrm{r}$ & + & + & . \\
\hline
\end{tabular}




\begin{tabular}{|c|c|c|c|c|c|c|c|c|c|c|c|c|c|c|c|}
\hline Relevé number & 1 & 2 & 3 & 4 & 5 & 6 & 7 & 8 & 9 & 10 & 11 & 12 & 13 & 14 & 15 \\
\hline Astrantia major & . & . & . & . & . & . & . & . & . & . & . & $\mathrm{r}$ & & . & $\mathrm{r}$ \\
\hline Bellidiastrum michelii & . & . & . & . & . & . & . & . & . & . & . & . & 2.2 & 1.2 & . \\
\hline Salix retusa & . & . & . & . & . & . & . & . & . & . & . & . & 1.2 & 2.2 & . \\
\hline $\begin{array}{l}\text { Carex sempervirens } \\
\text { subsp. tatrorum }\end{array}$ & . & . & . & . & . & . & $\cdot$ & . & . & . & . & . & +.2 & 2.2 & . \\
\hline Salix reticulata & . & . & . & . & . & . & . & . & . & . & . & . & + & 1.2 & . \\
\hline Pedicularis verticillata & . & . & . & . & . & . & . & . & . & . & . & . & + & 1.2 & . \\
\hline Androsace chamaejasme & . & . & . & . & . & . & . & . & . & . & . & . & +.2 & + & . \\
\hline Moehringia muscosa & . & . & . & . & . & . & . & . & . & . & . & . & +.2 & +.2 & . \\
\hline Poa alpina & . & . & . & . & . & . & . & . & . & . & . & . & $\mathrm{r}$ & + & . \\
\hline Erysimum sp. & . & . & . & . & . & . & . & . & . & . & . & . & $\mathrm{r}$ & $\mathrm{r}$ & . \\
\hline Selaginella selaginoides & . & . & . & . & . & . & . & . & . & . & . & . & $\mathrm{r}$ & $\mathrm{r}$ & . \\
\hline Moneses uniflora & . & . & . & . & . & . & . & . & . & . & . & . & $\mathrm{r}$ & $\mathrm{r}$ & . \\
\hline Cirsium erisithales & . & . & . & . & . & . & . & . & . & . & . & . & . & $\mathrm{r}$ & $\mathrm{r}$ \\
\hline Carex firma & . & . & . & . & . & . & . & . & . & . & . & . & $\mathrm{r}$ & + & . \\
\hline Crepis jacquinii & . & . & . & . & . & . & . & . & . & . & . & . & $\mathrm{r}$ & + & . \\
\hline Melampyrum sylvaticum & . & . & . & . & . & . & . & . & . & . & . & . & . & 2.3 & + \\
\hline Phyteuma orbiculare & . & . & . & . & . & . & . & . & . & . & . & . & . & 1.1 & + \\
\hline Saxifraga paniculata & . & . & . & . & . & . & . & . & $\mathrm{r}$ & . & +.2 & . & $\mathrm{r}$ & + & . \\
\hline Festuca versicolor & . & . & . & . & . & . & . & . & $\mathrm{r}$ & . & 2.2 & . & + & + & . \\
\hline Soldanella carpatica & . & . & . & + & . & . & . & . & . & . & + & . & 1.1 & + & . \\
\hline Rhodiola rosea & . & . & . & . & . & $\mathrm{r}$ & . & . & $\mathrm{r}$ & . & $\mathrm{r}$ & + & . & + & . \\
\hline Bistorta vivipara & . & . & . & $\mathrm{r}$ & . & . & . & . & . & . & . & . & + & + & . \\
\hline $\begin{array}{l}\text { Differential species for } t \\
\text { associations }\end{array}$ & & & & & & & & & & & & & & & \\
\hline Viola biflora & . & . & . & +.2 & +.2 & +.2 & . & 1.2 & 1.2 & . & . & + & . & . & . \\
\hline Ranunculus platanifolius & . & . & . & $\mathrm{r}$ & + & $\mathrm{r}$ & 1.1 & . & $\mathrm{r}$ & + & . & . & 1.1 & . & . \\
\hline Adenostyles alliariae & . & . & . & 1.2 & 2.3 & 1.2 & 1.2 & 4.4 & 3.3 & 2.2 & $\mathrm{r}$ & + & $\mathrm{r}$ & . & $\mathrm{r}$ \\
\hline Sesleria tatrae & . & . & . & +.2 & . & 1.2 & . & . & . & . & + & + & 2.2 & 2.2 & +.2 \\
\hline Cystopteris fragilis & . & . & . & . & $\mathrm{r}$ & . & . & + & + & $\mathrm{r}$ & 1.2 & $\mathrm{r}$ & $\mathrm{r}$ & $\mathrm{r}$ & $\mathrm{r}$ \\
\hline $\begin{array}{l}\text { Differential species of th } \\
\text { alliance Calamagrostio } v \\
\text { Pinion cembrae } \\
\text { Valeriana tripteris }\end{array}$ & . &. & . & +2 & 1.2 & + & + & 2.2 & + & 1.2 & + & 22 & +2 & 23 & 12 \\
\hline
\end{tabular}




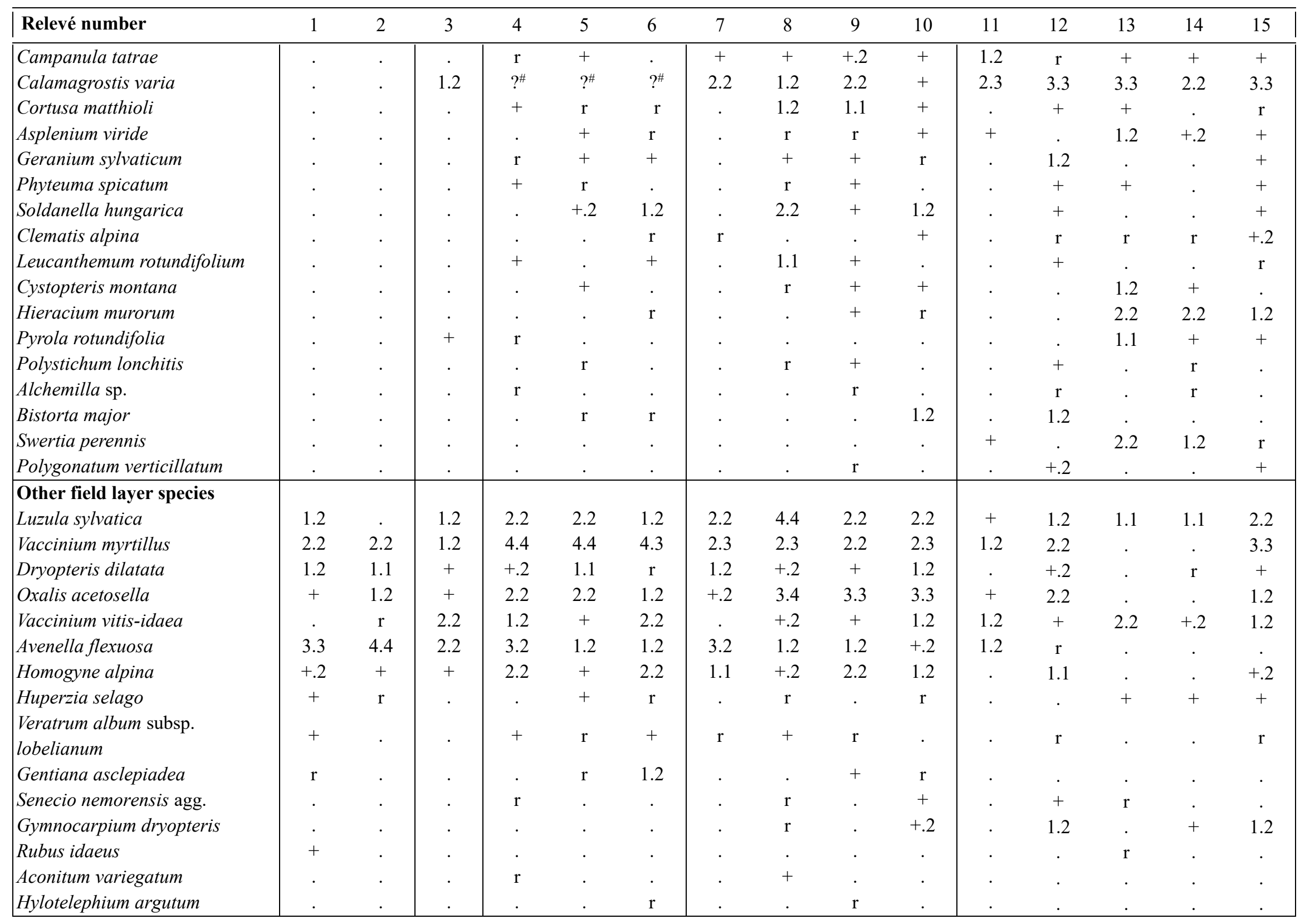




\begin{tabular}{|c|c|c|c|c|c|c|c|c|c|c|c|c|c|c|c|}
\hline Relevé number & 1 & 2 & 3 & 4 & 5 & 6 & 7 & 8 & 9 & 10 & 11 & 12 & 13 & 14 & 15 \\
\hline Saxifraga wahlenbergii & . & . & . & . & . & . & . & $\mathrm{r}$ & . & . & . & . & + & . & . \\
\hline Cicerbita alpina & . & . & . & . & . & . & . & . & 1.2 & . & . & 1.2 & . & . & . \\
\hline Geum rivale & . & . & . & . & . & . & . & . & $\mathrm{r}$ & . & . & + & . & . & . \\
\hline
\end{tabular}

\# Calamagrostis varia admixed in unspecified abundance.

\section{Selected field layer species in one relevé only:}

Rel. 2: Rubus sp. r.; Rel. 4: Potentilla aurea r.; Rel. 5: Athyrium filix-femina r, Galeobdolon luteum r.; Rel. 6: Oreogeum montanum r, Potentilla sp. r, Senecio subalpinus r.; Rel.

7: Arabis alpina +, Cystopteris sp. +, Galeopsis sp. +; Rel. 10: Cardaminopsis arenosa r.; Rel. 11: Draba tomentosa r.; Rel. 12: Cyanus mollis +, Ligusticum mutellina +.; Rel. 13: Ranunculus sp. +, Scabiosa lucida +, Cerastium arvense subsp. glandulosum r, Parnassia palustris r.; Rel. 14: Dryas octopetala +.2, Tofieldia calyculata + ,

Helianthemum grandiflorum r, Astragalus norvegicus r, Pinguicula alpina r.; Rel. 15: Galium anisophyllon r.

\section{Relevé localities:}

Rel. 1: High Tatras, Kolová dolina, slope of Jahnence Mt., 49¹3'34" N, 20¹1'13" E, bedrock: quartzose sandstones, quartzites, relief: bouldered consolidated talus on moderately steep slope, soil: lithosol (lithic leptosol), tree height: 5-15 m, tree trunk diameter: 8-70 cm, age of trees: 30-300 yrs, 29. 7. 1995, P. Barančok.

Rel. 2: High Tatras, Kolová dolina, slope of Jahnence Mt., 49¹3'35" N, 20¹1'14" E, bedrock: transitional area between (1) variegated shales, sandstones, marly shales, limestones and (2) quartzites, relief: steep bouldery slope, soil: lithosol (lithic leptosol), tree height: up to $20 \mathrm{~m}$, tree trunk diameter: up to $45 \mathrm{~cm}$, age of trees: up to 200 yrs, 29 . 7. 1995, P. Barančok.

Rel. 3: High Tatras, Kolová dolina, Úplaz Mt., 49 $13^{\prime} 44^{\prime \prime}$ N, $20^{\circ} 10^{\prime} 45^{\prime \prime}$ E, bedrock: massive organogene limestones, relief: top of a cliff, tree height: 5-15 m, tree trunk diameter: 10-40 cm, age of trees: 50-200 yrs, 12. 7. 1995, P. Barančok.

Rel. 4: High Tatras, Jahnence Mt., Kolová dolina - right slope of the valley above the forest path, 49²13'40" N, 20¹1'09" E, bedrock: transitional area between (1) dark partly dolomitized limestones, wormy limestones and (2) variegated shales, sandstones, marly shales, relief: rugged slope, tree height: up to 20 m, tree trunk diameter: Pinus 25-40 cm, Picea 15-25 cm, age of trees: Pinus up to $200 \mathrm{yrs}$, Picea $60 \mathrm{yrs}, 8.7 .1995$, P. Barančok.

Rel. 5: High Tatras, Kolová dolina, right slope, $100 \mathrm{~m}$ above the forest path, 49¹3'39" N, 20¹1'04" E, bedrock: variegated shales, sandstones, marly shales, limestones, relief: steep slope rugged with erosion furrows, tree height: up to $20 \mathrm{~m}$, tree trunk diameter: Pinus up to $50 \mathrm{~cm}$, Picea up to $25-30 \mathrm{~cm}$, age of trees: Pinus $100-300$ yrs, Picea up to 100 yrs, 9. 7. 1995, P. Barančok.

Rel. 6: High Tatras, Kolová dolina, right slope, 49¹3'38" N, 20¹1'07" E, bedrock: (1) variegated shales, sandstones, marly shales, limestones and (2) quartzites, relief: rugged ridge gradually protruding of the slope, inclination: ridge $10-15^{\circ}$ (downwards up to $40^{\circ}$ ), slope in general $60-70^{\circ}$, aspect: ridge $\mathrm{W}$, its slopes $\mathrm{NW}$ and $\mathrm{SW}$, tree height: up to 20 (25) m, tree trunk diameter: Pinus 40-70 cm, Picea 20-30 cm, age of trees: Pinus 150-300 yrs., Picea 80 (100) yrs, 9. 7. 1995, P. Barančok.

Rel. 7: High Tatras, Jahňací úplaz, ridge between the Kolová dolina Valley and the Zadné Med’odoly Valley, 49¹3'50" N, 20¹1'19" E, bedrock: dark partly dolomitized limestones, wormy limestones, relief: rocky rib, tree height: 15-25 m, tree trunk diameter: Pinus 25-60 cm, Picea 10-25 cm, age of trees: Pinus 80-250 yrs, Picea up to 50 yrs, 20. 8. 1994, P. Barančok.

Rel. 8: High Tatras, Jahnence Mt., Jahňací úplaz ridge, 49¹3'48" N, 20¹1'15" E, bedrock: dark partly dolomitized limestones, wormy limestones, relief: undulated slope, tree height: Pinus up to $20 \mathrm{~m}$, Picea $30 \mathrm{~m}$, tree trunk diameter: in average up to $45 \mathrm{~cm}$, age of trees: Pinus 100-250 yrs, Picea 100-140 yrs, 8. 7. 1995, P. Barančok.

Rel. 9: High Tatras, Jahnence Mt., Jahňací úplaz ridge, 49 $13^{\prime} 46^{\prime \prime}$ N, 20¹1'16" E, bedrock: dark partly dolomitized limestones, wormy limestones, relief: rocky rib, 
inclination: slope in general $40-45^{\circ}$, rocky rib $15^{\circ}$, southern wall of the rocky rib $80^{\circ}$, aspect: W for general slope and also for the rocky rib, tree height: Pinus $15-25 \mathrm{~m}$, tree trunk diameter: 20-55 cm, age of trees: Pinus 80-300 yrs, Picea up to $60 \mathrm{yrs}, 8.7 .1995$, P. Barančok.

Rel. 10: High Tatras, Kolová dolina, slope of Úplaz Mt., 49 $13^{\prime} 47^{\prime \prime}$ N, $20^{\circ} 10^{\prime} 44^{\prime \prime}$ E, bedrock: massive organogene limestones, relief: steep slope moderately rugged, tree height: 20-25 (30) m, tree trunk diameter: 15-35 (40) cm, age of trees: 80-150 yrs, 12. 7. 1995, P. Barančok.

Rel. 11: High Tatras, Jahnence Mt., Kolová dolina - right slope of the valley above the forest path, 49 $13^{\prime} 42^{\prime \prime} \mathrm{N}, 20^{\circ} 11^{\prime} 13^{\prime \prime} \mathrm{E}$, bedrock: dark partly dolomitized limestones, wormy limestones, relief: a rocky rib on steep slope, with exposed bedrock stones, tree height: $10 \mathrm{~m}$, tree trunk diameter: up to $25 \mathrm{~cm}$, age of trees: up to $100 \mathrm{yrs}, 8.7 .1995$, P. Barančok.

Rel. 12: Belianske Tatry Mts, Monkova dolina, right slope of the valley, 49 $144^{\prime} 46^{\prime \prime} \mathrm{N}, 20^{\circ} 13^{\prime} 46^{\prime \prime} \mathrm{E}$, bedrock: light-coloured pelitic limestones and spotted marls, relief: steep slope, tree height: 10-20 (25) m, tree trunk diameter: 20-45 cm, age of trees: 80-150 (200) yrs, 11. 7. 1995, P. Barančok.

Rel. 13: Belianske Tatry Mts, Pod Sikou Valley, north-oriented slopes of the rock formation Veký Cosek, 49¹4'39" N, 20¹4'05" E, bedrock: (1) light-coloured pelitic limestones, spotted marls and (2) Murán̆ limestones, relief: rugged steep slope below the rock formation, soil: lithosol (lithic leptosol), tree height: 10-15 up to 20 m, tree trunk diameter: 10-70 cm, age of trees: 40-250 yrs, 26. 7. 1995, P. Barančok.

Rel. 14: Belianske Tatry Mts, Pod Sikou Valley, north-oriented slopes of the rock formation Veký Č́sek, 49¹4'40" N, 20¹4'07" E, bedrock: (1) light-coloured pelitic limestones, spotted marls and (2) Murán̆ limestones, relief: rugged steep slope below the rock formation, soil: lithosol (lithic leptosol), tree height: up to 20 m, tree trunk diameter: 10-50 cm, age of trees: Pinus 40-200 yrs, 26. 7. 1995, P. Barančok.

Rel. 15: Belianske Tatry Mts, Pod Sikou Valley, north-oriented slopes of the rock formation Vel'ký Čosek, 49¹4'43" N, 20¹4'13" E, bedrock: (1) light-coloured pelitic limestones, spotted marls and (2) Muráň limestones, relief: steep slope rugged with rock cuts, soil: lithosol (lithic leptosol), tree height: 25-30 m, tree trunk diameter: 15-50 cm, age of trees: 60-250 yrs, 26. 7. 1995, P. Barančok. 
By contrast, the subassociation Cystopterido montanae-Pinetum cembrae calamagrostietosum arundinaceae (Zięba et al. 2018) P. Kučera 2019 [17] is characterized by the constant presence and higher cover of Calamagrostis arudinacea, as well as by the occurrence of Carex sempervirens subsp. tatrorum, Saxifraga paniculata, Astrantia major, etc. (cf. [39]: suppl. S3). Before the start of human exploitation of the high elevations of the Tatra Mountains, forest stands of the latter subassociation were probably the most frequent Arolla pine community of the calcareous regions from the west (Sivý vrch Mt.) to the east (Belianske Tatry Mts). It developed also at sites with deeper and well-drained soils where ecological influence of more densely growing Pinus cembra trees (shade, slowly decaying fallen long needles covering surface and less rainfall water blocked by the tree crowns) induces development of mixed acidophytic-calciphytic species composition.

2.3. Primulo elatioris-Pinetum cembrae ass. nov. hoc loco (Tab. 1, rels. 7-10)

Nomenclatural type: tab. 1, rel. 8, holotypus hoc loco

This Arolla pine community is developed on sites with the most favourable water regime; habitats are mesic and mesotrophic. Physiognomy of the field layer is determined by Adenostyles alliariae (usually a dominant), Luzula sylvatica and Oxalis acetosella. Present also are other tall herbs such as Cicerbita alpina, Doronicum austriacum, Leucanthemum rotundifolium and Veratrum album subsp. lobelianum. Into this association is concentrated the occurrence of species with higher demands on habitat humidity, i.e. Chrysosplenium alternifolium, Stellaria nemorum and Chaerophyllum hirsutum; in some localities is present also Sphagnum sp.

Of characteristic occurrence are calcicoles - Asplenium viride, Calamagrostis varia, Cortusa matthioli, Cystopteris montana etc.; important components of the association are nutrientdemanding species, e.g. Geranium sylvaticum, Valeriana tripteris, Viola biflora and Thalictrum aquilegiifolium.

Constantly present are the acidophytes Vaccinium myrtillus (cover over $5 \%$ ), V. vitisidaea, Avenella flexuosa; frequent also are other common species of high montane forests (Dryopteris dilatata, Homogyne alpina, Gentiana asclepiadea).

Up to the present time, this association was recorded only within the Kolová dolina Valley of the High Tatras.

\subsection{Seslerio tatrae-Pinetum cembrae P. Kučera 2017 (Tab. 1, rels. 11-15)}

This plant association occupies habitats on calcareous rocks which possess the highest number of calcicoles among the communities of the alliance Calamagrostio variae-Pinion cembrae. Calamagrostis varia is the dominant species of the field layer. Constantly present are Valeriana tripteris, Sesleria tatrae, Luzula sylvatica, Campanula tatrae, Clematis alpina, Cortusa matthioli, Phyteuma spicatum, Dryopteris dilatata, Vaccinium vitis-idaea, also Adenostyles alliariae (but only at low abundance) and many other species. Ecologically special habitat is expressed with occurrence of species such as Androsace chamaejasme, Bartsia alpina, Hedysarum hedysaroides, Ranunculus alpestris, Pedicularis verticillata, Salix reticulata and S. retusa, as well as Carex firma, Festuca versicolor, Rhodiola rosea and Saxifraga paniculata.

The presence and abundance of acidophytes (Avenella flexuosa, Vaccinium myrtillus) is usually reduced; absent or infrequent also are the species Gentiana aslepidaea, Senecio 
nemorensis agg. and Veratrum album subsp. lobelianum, which are more common in mesic habitats.

The association Seslerio tatrae-Pinetum cembrae was noted mainly in the Belianske Tatry Mts where its occurrence was documented also by Kanka [12: tab. 11, rel. 5]. Zięba et al. [39: suppl. S3, rel. 32] published one record of this community from the High Tatras.

Acknowledgements: I would like to express my sincere thanks to Prof. Bohdan Juráni (Bratislava) for his help with the English names of soil units. I would also like to thank to the editorial office for the corrections of my English. This study was supported by the Slovak grant agency VEGA, projects No. 2/0119/19 and No. 2/0132/18.

\section{REFERENCES}

1. Barančok, P., 2002, Limbovo-smrekové porasty východnej časti Tatier (Belianske Tatry a východná čast' Vysokých Tatier) [Arolla pine-Norway spruce stands of the eastern part of the Tatras (Belianske Tatry Mts and eastern part of the High Tatras)]. In: Kubíček, F., Kanka, R., Kollár, J. et Barančok, P. (eds.), Ekológia a produktivita bylinnej vrstvy lesných ekosystémov: Zborník z medzinárodného odborného seminára, konaného v dňoch 13.-15. 11. 2001 na Výskumnej stanice ÚKE SAV vo Východnej. Bratislava: 120-129.

2. Barančok, P., Varšavová, M., 1995, Spread and phytocoenological characteristic of ceder pine-spruce forests of Bielovodská dolina Valley in the Vysoké Tatry Mountains, Ekológia (Bratislava), 14 (Supplement 2/1995): 35-51.

3. Barančok, P., Varšavová, M., 1998, Influence of exogenous factors on the distribution of ceder pine-spruce forests and dwarf pine forests in the Belianske Tatry Mts., Ekológia (Bratislava), 17 (3): 242-254.

4. Braun-Blanquet, J., 1951, Pflanzensoziologie. Grundzüge der Vegetationskunde. 2., umgearb. vermehr. Aufl. Springer, Wien.

5. Hennekens, S. M. c1998-2020, Turboveg for Windows. International single user version [disk]. Ver. 2.149a. (c) 1998-2020 S. M. Hennekens, Wageningen. Comprehensive database management system designed for the storage, selection, and export of vegetation data (relevés). Available on internet: $<\mathrm{http}: / /$ www.synbiosys.alterra.nl/turboveg/>.

6. Hennekens, S.M., Schaminée, J.H.J., 2001, Turboveg, a comprehensive database management system for vegetation data, Journal of Vegetation Science, 12: 589-591. DOI: 10.2307/3237010

7. Hołub-Pacewiczowa, Z., 1931, Osadnictwo pasterskie i wędrówki w Tatrach i na Podtatrzu [L’habitat et les migrations pastorales dans les Tatra et dans la région subtatrique], Prace Komisji geograficznej, 1: XX + 508 p., fig. and mp. app.

8. Jamnický, J., 1981, Rozšírenie a stav limby (Pinus cembra L.) v Západných Karpatoch [Distribution and conditions of stone-pine (Pinus cembra L.) in the Western Carpathians], Zborník prác o Tatranskom národnom parku, 22: 5-29.

9. Jarolímek, I., Šibík, J., Hegedüšová, K., Janišová, M., Kliment, J., Kučera, P., Májeková, J., Michálková, D., Sadloňová, J., Šibíková, I., Škodová, I., Uhliřrová, J., Ujházy, K., Ujházyová, M., Valachovič, M., Zaliberová, M., 2008, A list of vegetation units of Slovakia. In: Jarolímek, I., Šibík, J. (eds.) et al., Diagnostic, constant and dominant species of the higher vegetation units of Slovakia. VEDA, vydavatel'stvo Slovenskej akadémie vied, Bratislava: 295-329.

10. Jasík, M., Dítě, D., 2016, Súčasné poznatky o rozšírení linnéovky severskej (Linnaea borealis L.) na Slovensku. [Current knowledge about the distribution of twinflower (Linnaea borealis L.) in Slovakia], Acta Carpathica Occidentalis, 7: 9-14.

11. Jasík, M., Dítě, D., Eliáš, P., jun., 2014, Linnaea borealis (linnéovka severná) na Slovensku [Linnaea borealis (twinflower) in Slovakia], Bulletin Slovenskej botanickej spoločnosti, 36 (1): 49-56.

12. Kanka, R., 2008a, Lesy Belianskych Tatier [Forests of the Belianske Tatry Mts], VEDA, vydavatel'stvo Slovenskej akadémie vied, Bratislava.

13. Kanka, R., 2008b, Limbové a limbovo-smrekové lesné spoločenstvá Belianskych Tatier [Swiss stone pine and Swiss stone pine-spruce forest communities in the Belianske Tatry mountains], Phytopedon, 7 (1-2): 1825 . 
14. Kolb, E., Kohlpaintner, M., 2018, Tangel humus forms - genesis and co-evolution with vegetation, Applied Soil Ecology, 123: 622-626. DOI: 10.1016/j.apsoil.2017.09.040

15. Kučera, P., 2012, Vegetačný stupeň smrečín v Západných Karpatoch - rozšírenie a spoločenstvá: Spis so zvláśtnym zretelom na pohorie Vel'ká Fatra [Norway spruce altitudinal vegetation zone in the Western Carpathians - distribution and communities], Botanická záhrada UK v Bratislave, pracovisko Blatnica, Blatnica.

16. Kučera, P., 2017, Two groups of Pinus cembra forest communities in the Tatras, Acta Botanica Hungarica, 59 (3-4): 389-425. DOI: $10.1556 / 034.59 .2017 .3-4.7$

17. Kučera, P., 2019, Pinus cembra communities in the Tatras - comments to the study of Zięba et al. Tuexenia, 39: 161-180. DOI: 10.14471/2019.39.013

18. Kučera, P., 2021a, On the pseudonymous syntaxonomical application of the order Athyrio-Piceetalia Hadač 1962 , In red.

19. Kučera, P., 2021b, Natural calcareous Norway spruce woodlands in Slovakia and their syntaxonomical classification, Hacquetia, In red.

20. Kukla, J., Kuklová, M., Schieber, B., 2004, Responses of some herbs to different ecological conditions in spruce ecosystems of the Bielovodská dolina valley, Ekológia (Bratislava), 23 (3): 252-269.

21. Marhold, K. [ed.], Goliašová, K., Hegedüšová, Z., Hodálová, I., Jurkovičová, V., Kmetová, E., Letz, R., Michalková, E., Mráz, P., Peniašteková, M., Šípošová, H., Ťavoda, O. et al., 1998, Paprad’orasty a semenné rastliny [Ferns and Flowering Plants]. In: Marhold, K., Hindák, F. (eds.) et al. Zoznam nižšich a vyššich rastlin Slovenska [Checklist of non-vascular and vascular plants of Slovakia], VEDA, vydavatel'stvo Slovenskej akadémie vied, Bratislava: 333-687.

22. Matuszkiewicz, J., 1977, Przegląd fitosocjologiczny zbiorowisk leśnych Polski. Cz. 4. Bory świerkowe i jodłowe [Phytosociological review of the forest communities of Poland. Part 4. Spruce and fir forests], Phytocoenosis, 6/3: 151-226, tab. app.

23. Matuszkiewicz, J.M., 2002, Zespoly leśne Polski [Forest communities of Poland], 1st ed., reprint. Wydawnictwo Naukowe PWN, Warszawa.

24. Matuszkiewicz, W., 1981, Przewodnik do oznaczania zbiorowisk roślinnych Polski [Guide for the determination of plant communities of Poland], 1st ed. Państwowe Wydawnictwo Naukowe, Warszawa.

25. Matuszkiewicz, W., 1984, Die Karte der potenziellen natürlichen Vegetation von Polen, Braun-Blanquetia, 1: 100 p., mp. app.

26. Matuszkiewicz, W., 2014, Przewodnik do oznaczania zbiorowisk roślinnych Polski [Guide for the determination of plant communities of Poland], New ed. (3rd changed and appended) - 9th reprint. Wydawnictwo Naukowe PWN, Warszawa.

27. Matuszkiewicz, W., Matuszkiewicz, J.M., 1996, Przegląd fitosocjologiczny zbiorowisk leśnych Polski. (Synteza) [Pflanzensoziologische Übersicht der Waldgesellschaften von Polen (Synthese)]], Phytoceonosis (N. S.), 8 (Semin. geobot. 3): 3-79.

28. Morfogenetický klasifikačný systém pôd Slovenska: bazálna referenčná taxonómia [Morphogenetic soil clasification system of Slovakia], 2014, 2nd corr. ed. Výskumný ústav pôdoznalectva a ochrany pôdy NPPC, Bratislava.

29. Myczkowski, S., 1969, Limba Pinus cembra L. - wysokogórskie drzewo lasu tatrzańskiego [Stone pine (Pinus cembra L.) - an alpine tree of the Tatra forests], Zborník prác o Tatranskom národnom parku, 11: 99-138.

30. Myczkowski, S., Lesiński, J., 1974, Rozsiedlenie rodzimych gatunków drzew tatrzańskich [Distribution of native tree species in the Tatra Mountains], Studia Ośrodka Dokumentacji Fizjograficznej, III: 13-70.

31. Nemčok, J. (ed.), Bezák, V., Biely, A., Gorek, A., Gross, P., Halouzka, R., Janák, M., Kahan, Š., Kotański, Z., Lefeld, J., Mello, J., Reichwalder, P., Raczkowski, W., Roniewicz, P., Ryka, W., Wieczorek, J., Zelman, J., 1993, Geologická mapa Tatier [Geological map of the Tatra Mountains], Geologický ústav Dionýza Štúra, Bratislava.

32. Pawłowski, B., Sokołowski, M., Wallisch, K., 1928, Die Pflanzenassoziationen des Tatra-Gebirges. VII. Teil. Die Pflanzenassoziationen und die Flora des Morskie Oko-Tales, Bulletin International de l'Académie Polonaise des Sciences et des Lettres, Classe des Sciences Mathématiques et Naturelles, Série B.: Sciences Naturelles, 1928 (Suppl. II.): 205-272, tab., fig. and mp. app.

33. Samek, V., Jančařík, V., Kriesl, A., Materna, J., 1957, Lesní společenstva severního úbočí Vysokých Tater 
(Část I. Javorová dolina) [Die Waldgesellschaften der Nordabhänge der Hohen Tatra (Teil I. Javorinatal)]. Lesnicky časopis, III (1): 3-38, tab. app.

34. Šibík, J., 2012, Slovak Vegetation Database. In: Dengler, J., Oldeland, J., Jansen, F., Chytrý, M., Ewald, J., Finckh, M., Glöckler, F., Lopez-Gonzalez, G., Peet, R. K., Schaminée, J. H. J. (eds.): Vegetation databases for the 21st century, Biodiversity \& Ecology, 4: 429 [Short Database Report]. DOI: 10.7809/b-e.00216.

35. Theurillat, J.-P., Willner, W., Fernández-González, F., Bültmann, H., Čarni, A., Gigante, D., Mucina, L., Weber, H., 2020, International Code of Phytosociological Nomenclature, 4th edition, Applied Vegetation Science, DOI: $10.1111 /$ avsc. 12491

36. Tichý, L., c1998-2020, JUICE [disk], Ver. 7.1.25. @ 1998-2020 L. Tichý, Brno, Program for analysis and classification of phytosociological tables and other quantitative ecological data sets. Available on internet: $<\mathrm{http}: / /$ www.sci.muni.cz/botany/juice/>.

37. Tichý, L., 2002, JUICE, software for vegetation classification, Journal of Vegetation Science, 13: 451-453. DOI: 10.1111/j.1654-1103.2002.tb02069.x

38. Wojterska, M., Wojterski, T., Szwed, W., Pisaszyk, M., 2004, Spruce forests in the Roztoka Valley in the High Tatras. In: Brzeg, A., Wojterska, M. (eds), Coniferous forests vegetation - differentiation, dynamics and transformations, Wydawnictwo Naukowe Uniwerzitetu im. Adama Mickiewicza w Poznaniu, Poznań: $115-128$.

39. Zięba, A., Różański, W., Szwagrzyk, J., 2018, Syntaxononomy of relic Swiss stone pine (Pinus cembra) forests in the Tatra Mountains, Tuexenia, 38: 155-176. DOI: 10.14471/2018.38.004

40. Zlatník, A., 1970, Ekologicko-synekologický, cenologický a fytogeografický výskum na trvalých výskumných plochách [Ecologico-synecologic, coenologic and phytogeographic studies on permanent experimental plots], Zborník prác o Tatranskom národnom parku, 12: 79-152, tab. app.

\title{
CONTRIBUȚII LA CUNOAŞTEREA VARIABILITĂŢII PĂDURILOR DE PINUS CEMBRA DIN NORD-ESTUL MUNȚILOR TATRA
}

\begin{abstract}
(Rezumat)
Cunoașterea generală a variabilității sintaxonomice și de habitat a comunităților forestiere cu zâmbru (Pinus cembra) din cadrul Munților Tatra (Carpații de Vest) este încă insuficientă, din cauza accesibilității grele a locaţiilor și a defrișărilor făcute pentru a mări suprafața de pășune alpină. Pădurile de zâmbru au fost tradițional incluse în asociația Pino cembrae-Piceetum Myczkowski et Lesiński 1974, însă cercetări recente recunosc mai multe subunități. Relevee nepublicate din partea nord-estică a munților Tatra dovedesc existența mai multor tipuri de comunităţi de zâmbru, distincte din punct de vedere floristic și ecologic, precum: (1) păduri acide de Homogyno alpinae-Pinetum cembrae în habitate sărace în nutrienți pe substrat cuarțic și (2) păduri calcaroase cu puține specii Pyrolo rotundifoliae-Pinetum cembrae în situri cu un orizont de sol cu humus bine dezvoltat, Cystopterido montanae-Pinetum cembrae în habitate influențate atât de calcare, cât și de cuarț, Primulo elatioris-Pinetum cembrae legate de situri cu umiditate favorabilă și Seslerio tatrae-Pinetum cembrae cu cel mai pronunțat caracter calcaros.
\end{abstract}

Received: 25.02.2021; Accepted: 9.06.2021. 